\title{
Importancia del seguimiento de pacientes con cardiopatía isquémica en tratamiento con atorvastatina como prevención secundaria. Estudio piloto
}

\author{
F. DE LA IGLESIA MARTÍNEZ, S. PITA FERNÁNDEZ*, V. RAMOS POLLEDO, \\ C. PELLICER VÁZQUEZ, R. NICOLÁS MIGUEL, F. DIZ-LOIS MARTÍNEZ \\ Unidad de Corta Estancia Médica. *Unidad de Epidemiología Clínica y Bioestadística. \\ Complexo Hospitalario Juan Canalejo. A Coruña.
}

FOLLOW UP RELEVANCE IN THE SECONDARY PREVENTION OF ISCHEMIC HEART DISEASE WITH ATORVASTATIN. PILOT STUDY

\section{RESUMEN}

Objetivo: Determinar la importancia de la planificación del seguimiento en la prevención secundaria de la cardiopatía isquémica

Material y métodos: Diseño. Estudio prospectivo con grupo control concurrente y con asignación aleatoria balanceada.

Emplazamiento: Ambito ambulatorio.

Pacientes: 75 pacientes con cardiopatía isquémica que precisaron ingreso por síndrome agudo y en tratamiento con atorvastatina como prevención secundaria fueron divididos en dos grupos.

Intervenciones: El grupo experimental (40p) fue revisado cada 2 meses mediante examen físico, analítico, insistencia en dieta-ejercicio y ajuste de dosis de estatina. El grupo control (35p) fue remitido a su seguimiento habitual.

Resultados: El 88\% fueron varones. La media de edad fue de $63.7 \pm$ 10 años. En el grupo control hubo una mayor proporción de pacientes con HTA y toma de IECAs. En el resto de las características basales, porcentajes de infarto, angioplastia o cirugía y valores analíticos iniciales no existieron diferencias significativas. A los 6 meses la reducción de colesterol total, colesterol-LDL y triglicéridos fue mayor en el grupo experimental que en el control ( 33 vs. 24,34 vs. 26 y 51 vs. $24 \mathrm{mg} / \mathrm{dl}$, respectivamente), aunque no alcanzó significación estadística. El porcentaje de reingresos fue menor en el grupo experimental $(7,3 \%$ vs. $19,4 \%, \mathrm{P}=$ $0.114)$. Falleció un paciente en cada grupo.

Conclusiones: Los resultados apuntan que la planificación del seguimiento de pacientes con cardiopatía isquémica tiene especial relevancia para acercarse a los objetivos recomendados en la prevención secundaria.

PALABRAS CLAVE: Cardiopatía isquémica. Prevención secundaria Planificación del seguimiento.

\section{ABSTRACT}

Objective: To determine the usefulness of different intensities of follow up when atorvastatin is used in the secondary prevention of ischemic heart disease.

Material and methods: Design. Prospective study with balanced ran domized allocation including a concurrent control group.

Setting: Ambulatory care.

Patients: 75 patients hospitalized because of acute coronary syndro mes were administered atorvastatin as secondary prevention of ischemic heart disease and randomized either to intensive folow up or to a control visit 6 months apart.

Interventions: The intensive folow up group (40 patients) was contro lled every 2 months as outpatients and a physical examen and lipid profile was done, enhancing diet and exercise and adjusting the statin dose. The control group was evaluated just once after a 6 six month period.

Results: $88 \%$ were males and the average age was $63.7 \pm 10$ year. Basal characteristics, myocardial infarction, revascularized procedures and initial chemistry and lipid profile were similar in both groups, alt hough there was a higher proportion of patients in the control group taking ACE. After 6 months total cholesterol, LDL colesterol and trigly cerides reduction was higher in the intensive follow up group (33 vs. 24 , 34 vs. 26 y 51 vs. $24 \mathrm{mg} / d \mathrm{l}$, respectively) without realising statistical sig nificance. Hospital readmissions were lesser in the intensive group (7.3\% vs. $19.4 \%, P=0.114)$. There was one death in each group.

Conclusions: Results show a tendency indicating that follow up pla nification is relevant to obtein the target lipid level recomended in the secondary prevention.

KEY WORDS: Ischemic heart disease. Secondary prevention. Follow up planification.

De la Iglesia Martínez F, Pita Fernández S, Ramos Polledo V, Pellicer Vázquez, C, Nicolás Miguel R, Diz-Lois Martínez. F. Importancia del seguimiento de pacientes con cardiopatía isquémica en tratamiento con atorvastatina como prevención secundaria. Estudio piloto. An Med Interna (Madrid) 2002; 19: 11-15.

\section{INTRODUCCIÓN}

Las enfermedades del aparato circulatorio originan casi el $40 \%$ de las defunciones en España. Las debidas a cardiopatía isquémica, que eran una de las más bajas de los países industrializados, están aumentando desde los años setenta (1).
El control de los factores de riesgo cardiovascular es fundamental para prevenirlas. Los tres principales factores de riesgo coronario con carácter independiente, gradual y con valor predictivo positivo son las dislipemias, el consumo de cigarrillos y la hipertensión arterial. Los estudios clínicos han demostrado el beneficio de reducir el colesterol-LDL en

Trabajo aceptado: 18 Octubre de 2001

Correspondencia: Fernando de la Iglesia Martínez. Avda. del Ejército, 16 - $9^{\circ}$ C. 15.006. A Coruña. 
pacientes con cardiopatía isquémica y se recomienda mantenerlo en valores inferiores a $100 \mathrm{mg} / \mathrm{dl}(1,2)$. Sin embargo, los pacientes que se incluyen en los ensayos clínicos y su seguimiento dista mucho de las condiciones en las que se realiza la atención médica habitual. En España el grupo PRESENCIAP ha demostrado deficiencias en cuanto al registro, control y prescripción de fármacos para la prevención secundaria de la cardiopatía isquémica en Atención Primaria (3).

El presente estudio piloto se diseñó con el objetivo de conocer si el realizar un seguimiento programado mejora el control lipídico en pacientes isquémicos frente al seguimiento habitual de estos pacientes en nuestra área.

\section{MATERIAL Y MÉTODOS}

Se realizó un estudio prospectivo y aleatorio con grupo control concurrente y con asignación aleatoria balanceada incluyendo a todos los pacientes que ingresaron consecutivamente en la Unidad de Corta Estancia Médica de nuestro Hospital entre el 1 de enero y el 30 de abril de 2000 con el diagnóstico principal de cardiopatía isquémica.

Se recogieron la edad, sexo, peso, talla, índice de masa corporal (IMC, en $\mathrm{kg} / \mathrm{m}^{2}$ ) y lugar de residencia, dividiendo esta variable en tres categorías según residiesen en A Coruña o a una distancia inferior o superior a $25 \mathrm{~km}$ de la misma.

Con respecto al nivel socio-económico se tuvieron en cuenta los estudios realizados por el paciente y los ingresos familiares anuales declarados por ellos. Se anotaron los antecedentes patológicos conocidos con especial referencia a los factores de riesgo. Se definió diabetes mellitus e hipertensión arterial cuando presentaban cifras superiores a 126 $\mathrm{mg} / \mathrm{dl}$ ó 140/90 $\mathrm{mmHg}$, respectivamente o bien recibían terapia específica. Se consideró fumador al que hubiese fumado más de 20 paquetes de cigarrillos en su vida o más de 1 cigarrillo/día o más de un puro/semana durante un año. Se denominó fumador activo al que mantenía el hábito regularmente durante al menos el último mes. Se definió obesidad si el IMC > 25. Se anotaron los fármacos que tomaban los pacientes.

El estudio incluyó a todo enfermo con cardiopatía isquémica diagnosticada "de novo" o ya conocida que precisó ingreso por síndrome agudo. Se cuantificó el número de pacientes con infarto, realización de angioplastia con/sin implantación de prótesis y sometidos a cirugía de revascularización en el episodio actual o previamente. Al ingreso se determinó colesterol total, fracciones (HDL y LDL) y triglicéridos. Se prescribió una única estatina, la atorvastatina, con coste-efectividad demostrada (4), para evitar el sesgo de incluir diferentes estatinas. La dosis inicial al alta hospitalaria fue de $10 \mathrm{mg} /$ día.

Los pacientes fueron divididos mediante asignación aleatoria en dos grupos. El grupo experimental fue revisado ambulatoriamente por uno de los autores cada 2 meses. En las revisiones se realizó exámen físico y analítico. Se insistió en la modificación de hábitos dietéticos y en el ejercicio, y se aumentó la dosis de atorvastatina si el médico lo consideraba necesario. El grupo control fue remitido a su Médico de Cabecera sin programar revisiones en nuestra consulta y a los 6 meses se les convocó por teléfono para acudir al día siguiente a nuestra Unidad donde se realizó revisión física y analítica, se anotaron las modificaciones en el tratamiento, el número de reingresos y sus causas.
La evaluación a los 6 meses entre ambos grupos se basó en comparar los valores de colesterol, triglicéridos, abandono de medicación, reingresos hospitalarios por la misma causa y fallecimientos. La metodología del estudio fue del tipo de "intención de tratar".

El método estadístico incluyó el análisis descriptivo de cada variable del estudio expresando las medidas de centralización y dispersión de las cuantitativas y el valor absoluto y porcentaje de las cualitativas. El test de Kolmogorov-Smirnov comprobó la normalidad de las primeras. En la comparación de las variables se empleó el T-test de Student, el U-test de Mann-Whitney, el test de Chi-cuadrado y el test exacto de Fischer, según procediese. El nivel de significación establecido en todos los análisis fue del $5 \%$.

\section{RESULTADOS}

De los 75 pacientes incluidos, 40 fueron asignados al grupo experimental. 63 enfermos ingresaron en nuestra Unidad procedentes de Urgencias y 12 desde la UCI-Coronaria.

Las características basales de ambos grupos fueron similares salvo una mayor proporción de enfermos con HTA y de toma de inhibidores de la ECA en el grupo control (Tabla I).

TABLA I CARACTERÍSTICAS BASALES DE LOS PACIENTES

\begin{tabular}{|c|c|c|c|}
\hline \multirow{2}{*}{\multicolumn{2}{|c|}{$\begin{array}{c}\text { Grupo Experimental (40) } \\
\text { M ediana (rango) n (\%) }\end{array}$}} & \multicolumn{2}{|c|}{ Grupo Control (35) } \\
\hline & & lediana (rango) n (\%) & $p$ \\
\hline \multicolumn{4}{|c|}{ Características generales } \\
\hline Edad, años & $64(42-82)$ & $63,5(39-84)$ & 0,609 \\
\hline Sexo & & & 0,368 \\
\hline Varón & $37(90,2)$ & $30(83,3)$ & \\
\hline M ujer & $4(9,8)$ & $6(16,7)$ & \\
\hline \multicolumn{3}{|c|}{ Localidad de residencia } & 0,819 \\
\hline A Coruña & $23(56,1)$ & $18(50,0)$ & \\
\hline$<25 \mathrm{~km}$ & $10(24,4)$ & $9(25,0)$ & \\
\hline$>25 \mathrm{~km}$ & $8(19,5)$ & $9(25,0)$ & \\
\hline
\end{tabular}

Nivel económico (Ingresos familiares anuales en pesetas)

$\begin{array}{lrr}<1,5 \text { millones } & 21(51,2) & 17(47,2) \\ 1,5-6 \text { millones } & 19(46,3) & 17(47,2) \\ >6 \text { millones } & 1(2,4) & 2(5,6)\end{array}$

Estudios realizados por el paciente

$\begin{array}{lrr}\text { No estudios } & 2(4,9) & 3(8,3) \\ \text { Primarios incompletos } & 16(39,0) & 16(44,4) \\ \text { Primarios completos } & 15(36,6) & 13(36,1) \\ \text { Secundarios } & 7(17,1) & 2(5,6) \\ \text { Universitarios } & 1(2,4) & 2(5,6)\end{array}$

IMC, $\mathrm{kg} / \mathrm{m}^{2} \quad 27,7(18-33) \quad 26,4(21-37)$

Estancia, días 4 (1-16) 4,5 (1-13) 
TABLA I (continuación)

\begin{tabular}{lrrr}
\hline Antecedentes personales & & & \\
\hline Claudicación intermitente & $6(14,6)$ & $7(19,4)$ & 0,573 \\
Diabetes & $8(19,5)$ & $5(13,9)$ & 0,511 \\
HTA & $12(29,3)$ & $23(63,9)$ & 0,002 \\
O besidad & $33(80,5)$ & $28(77,8)$ & 0,769 \\
Antecedentes familiares & $9(22,0)$ & $12(33,3)$ & 0,263 \\
Fumador & $30(73,2)$ & $24(66,7)$ & 0,533 \\
Fumador activo & $5(12,2)$ & $7(19,4)$ & 0,381 \\
& & & \\
\hline Tratamiento previo & & & \\
\hline Beta-bloqueantes & $16(39,0)$ & $8(22,2)$ & 0,112 \\
Calcio-antagonistas & $8(19,5)$ & $13(36,1)$ & 0,102 \\
IECAs & $3(7,3)$ & $9(25,0)$ & 0,032 \\
Nitritos & $21(51,2)$ & $15(41,7)$ & 0,401 \\
Antiagregante & $24(58,5)$ & $23(63,9)$ & 0,630 \\
Estatina & $15(36,6)$ & $12(33,3)$ & 0,765 \\
Fibrato & $2(4,9)$ & $2(5,6)$ & 0,893 \\
Resinas & 0 & 0 & \\
\hline n: número de pacientes $\%:$ porcentae; & & \\
\hline
\end{tabular}

$\mathrm{n}$ : número de pacientes; \%: porcentaje; $\mathrm{p}$ : nivel de significación; IM C: índice de masa corporal; HTA: hipertensión arterial; IECA: inhibidor de la enzima convertidora de angiotensina.

No hubo diferencias en cuanto a número de infartos, tratamientos intervencionistas ni en los valores iniciales de colesterol o triglicéridos (Tabla II).

La figura 1 recoge la diferencia de los valores analíticos al inicio y a los 6 meses del estudio, expresada como media \pm desviación estándar. La tasa de reducción de colesterol total, colesterol-HDL, colesterol-LDL y triglicéridos fue de 10,7, $20,5,17,3$ y $14,2 \%$ en el grupo control. Dichos porcentajes en el grupo experimental fueron de 15,2, 12,8, 23,1 y 30\%, respectivamente. Al final del estudio sólo 11 pacientes del grupo experimental $(27,5 \%)$ y 9 del grupo control $(25,7 \%)$ alcanzaron el objetivo de colesterol-LDL $<100 \mathrm{mg} / \mathrm{dl}$.

Tres pacientes del grupo experimental y siete del grupo control reingresaron por el mismo problema (7,3 vs. 19,4\%, $\mathrm{p}=0,114)$. Suspendieron el tratamiento con estatinas 6 enfermos del grupo experimental y 8 del grupo control, la mayoría por indicación médica (15 vs. $22,9 \%, \mathrm{p}=0,681)$.

Durante el seguimiento falleció un paciente del grupo experimental (accidente cerebrovascular agudo) y otro del grupo control (suicidio por depresión).

\section{DISCUSIÓN}

En primer lugar queremos destacar que al tratarse de un estudio piloto con un número pequeño de pacientes la interpretación de los resultados debe tomarse como orientativa, y aún reconociendo la limitación de las conclusiones por esta causa nos parece interesante comunicar los hallazgos obtenidos. Nuestro estudio pretendió una aproximación a la realidad de nuestra área y valorar la influencia de programar visitas periódicas en el control lipémico.
TABLA II

CARACTERÍSTICAS BASALES DE LOS PACIENTES

\begin{tabular}{lrrr}
\hline \multicolumn{2}{c}{ Grupo Experimental (40) } & \multicolumn{3}{c}{ Grupo Control (35) } \\
\hline \multicolumn{2}{c}{ M ediana (rango) $n(\%)$} & Mediana (rango) $n(\%)$ & $p$ \\
\hline \multicolumn{4}{l}{ Cardiopatía isquémica: caracterización } \\
\hline Infarto, antiguo o reciente & $20(48,8)$ & $15(41,7)$ & 0,531 \\
ACTP y/o Stent & $12(29,3)$ & $16(44,4)$ & 0,167 \\
Cirugía (By-pass) & $10(24,4)$ & $7(19,4)$ & 0,601 \\
Tto. intervencionista & $20(48,8)$ & $21(58,3)$ & 0,401
\end{tabular}

Valores analíticos al inicio, en $\mathrm{mg} / \mathrm{dl}$

\begin{tabular}{lrrr}
\hline Colesterol & $222(108-354)$ & $221,5(116-356)$ & 0,403 \\
Colesterol-HDL & $37(25-73)$ & $37,5(20-63)$ & 0,994 \\
Colesterol-LDL & $153(79-233)$ & $146,5(49-250)$ & 0,720 \\
Triglicéridos & $126(42-1313)$ & $146(76-522)$ & 0,984
\end{tabular}

Valores analíticos a los 6 meses, en $\mathrm{mg} / \mathrm{dl}$

\begin{tabular}{lrrr}
\hline Colesterol & $174(112-349)$ & $204(143-288)$ & 0,078 \\
Colesterol-HDL & $43(24-73)$ & $46(29-70)$ & 0,231 \\
Colesterol-LDL & $111.5(69-202)$ & $118,5(79-201)$ & 0,148 \\
Triglicéridos & $104,5(42-478)$ & $115(5-69)$ & 0,229 \\
\hline
\end{tabular}

n: número de pacientes; \% : porcentaje; P: nivel de significación; ACTP: angioplastia coronaria transluminal percutánea; Tto. intervencionista: tratamiento con ACTP y/o implantación de Stent y/o cirugía de by-pass; HDL: lipoproteína de alta densidad; LDL: lipoproteína de baja densidad.

Las características basales de nuestros dos grupos son similares, salvo por una mayor proporción de toma de IECAs debido a un mayor diagnóstico de hipertensión arterial en el grupo control. Esta diferencia la relacionamos con el pequeño tamaño muestral y por primar, al balancear la asignación de pacientes, que el número infartos y/o tratamientos intervencionistas fueran comparables. Nuestro estudio incluye angina inestable ya que la prevención secundaria con estatinas en este grupo de pacientes obtiene un beneficio a largo plazo similar al que se obtiene en los que sobreviven a un infarto de miocardio (5).

Los resultados del estudio PREVESE sobre la prevención secundaria del infarto de miocardio en España llamó la atención sobre la escasa prescripción de fármacos hipolipemiantes $(6,7 \%)$ en el momento del alta hospitalaria (6). Dicho estudio constató además que a los 6 meses no había modificaciones favorables de las concentraciones de colesterol total o colesterol-LDL y sólo una discreta mejoría del colesterol-HDL (6). Los pacientes de los dos grupos de nuestro estudio mejoraron significativamente su perfil lipídico a los 6 meses (Fig. 1); si bien, la cuantía de la reducción del colesterol total y del colesterol-LDL fue más baja que la señalada en otros estudios (4).

Fueron pocos los pacientes que alcanzaron a los 6 meses el nivel deseable de colesterol-LDL en ambos grupos. Estos resultados subóptimos concuerdan con lo publicado en la literatura. El estudio EUROASPIRE I, realizado entre 1995 y 1996 mostró que a los 6 meses de un ingreso hospitalario por motivos idénticos a los de nuestro estudio, el número de enfermos que presentaba niveles elevados de colesterol alcan- 

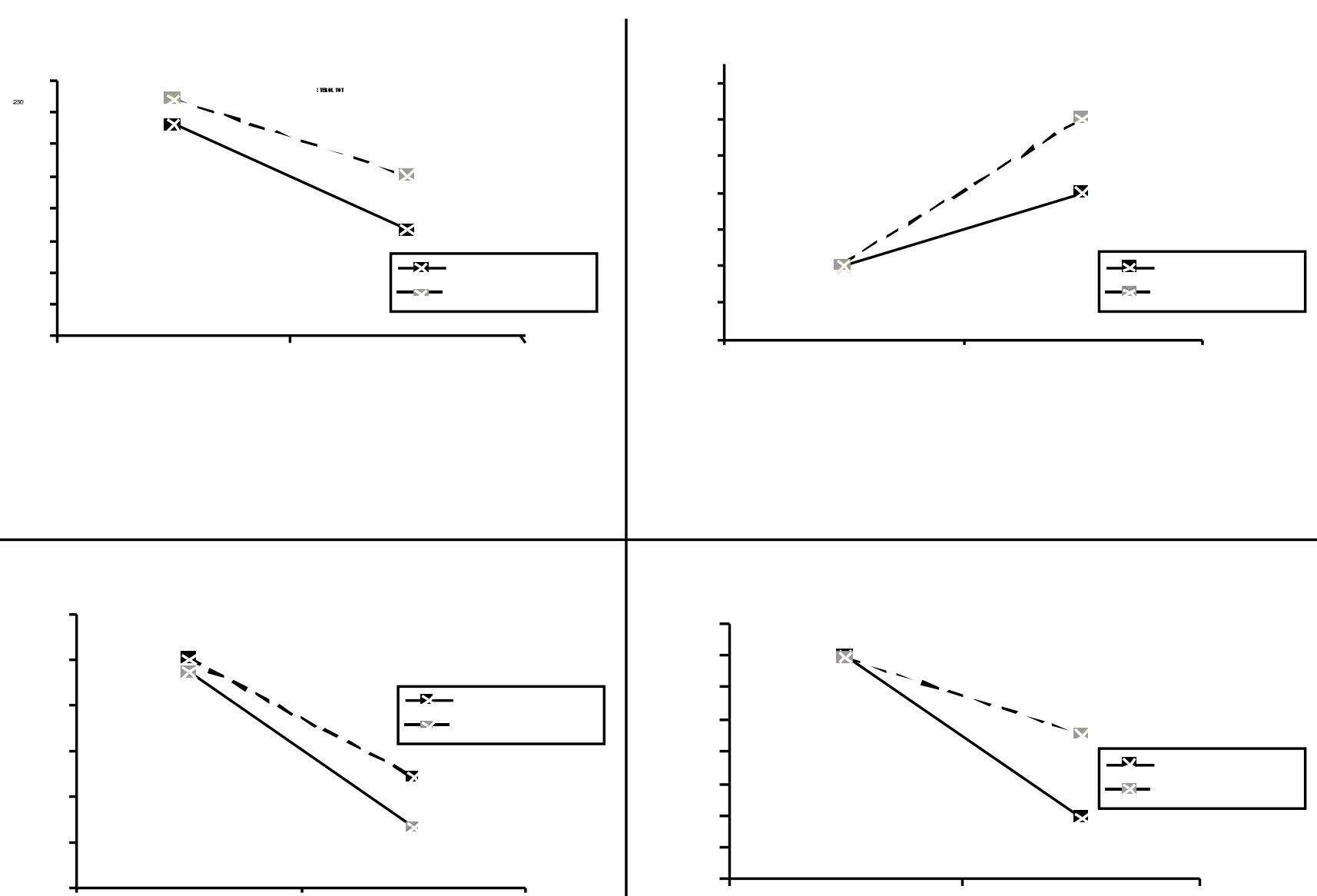

Fig. 1. Valor (media \pm desviación estándar) del colesterol y triglicéridos de los grupos experimental y control al inicio y a los 6 meses del estudio, expresados en $\mathrm{mg} / \mathrm{dl}$.

zaba el 86,2\% (7). Los resultados del EUROASPIRE II, realizado entre 1999 y 2000 , son mejores ya que la prevalencia de hipercolesterolemia disminuyó al 58,8\% (8). La situación en Estados Unidos no es muy diferente. En un reciente trabajo realizado en Boston el 64\% del total de los pacientes con enfermedad coronaria y necesidad de prevención secundaria estaban infratratados (9).

Aunque no objetivamos diferencias en el número de enfermos con colesterol-LDL óptimo a los 6 meses, los del grupo experimental obtuvieron una mayor reducción del colesterol total y LDL como lo muestra la pendiente de las rectas de la Figura 1. El que las diferencias no alcanzasen significación estadística lo relacionamos con el tamaño muestral. Probablemente si el número de participantes fuese mayor se cumplirían los requisitos matemáticos. El seguimiento programado tiene además la ventaja de conseguir un menor número de reingresos ya que el paciente comenta sus síntomas en las revisiones programadas y disminuye la frecuentación de los Servicios de Urgencias.
Holt demostró que aunque los pacientes intensifiquen sus chequeos de colesterol, las acciones emprendidas por los profesionales de la salud como consecuencia del resultado de los mismos son insuficientes (10). Este motivo y la necesidad de controlar los factores de riesgo con un nivel de exigencia elevado hace recomendar a los expertos que estos pacientes sean incluidos en programas específicos de prevención secundaria (1).

Consideramos que los resultados de nuestro estudio piloto apuntan en la dirección de que el seguimiento planificado de este grupo de enfermos es importante para obtener los objetivos recomendados, y estamos convencidos que los Médicos de Atención Primaria deben ser los principales protagonistas en esta estrategia.

\section{FINANCIACIÓN}

El presente estudio fue financiado, parcialmente, con una bolsa de investigación de Laboratorios Pfizer, S.A. 


\section{Bibliografía}

1. Plaza Pérez I, Villar Álvarez F, Mata López P, Pérez Jiménez F, Maiquez Galán A, Casasnovas Lenguas JA et al. Control de la colesterolemia en España, 2000. Un instrumento para la prevención cardiovascular. Rev Esp Cardiol 2000; 53: 815-837.

2. Sacks FM, Pfeffer MA, Moye LA et al. The effect of pravastatin on coronary events after myocardial infarction in patients with average cholesterol levels. Cholesterol and Recurrent Events Trial investigators. N Engl J Med 1996; 335: 1001-1009.

3. Grupo PRESENCIAP. Estudio de la prevención secundaria de la cardiopatía isquémica en atención primaria (PRESENCIAP). Aten Primaria $2001 ; 27: 29-32$

4. Tárraga López PJ, Celada Rodriguez A, Cerdán Oliver M, Solera Albero J, Ocaña López JM, de Miguel Clavé J. Análisis coste-efectividad de atorvastatina frente a simvastatina como tratamiento hipolipemiante en pacientes hipercolesterolémicos en atención primaria. Aten Primaria 2001; 27: 18-24.

5. Tonkin AM, Colquhoun D, Emberson J, Hague W, Keech A, Lane G et al. Effects of pravastatin in 3260 patients with unstable angina: results from LIPID study. Lancet 2000; 356: 1871-1875.

6. De Velasco JA, Cosín J, López Sendón JL, de Teresa E, de Oya M, Carrasco JL et al. La prevención secundaria del infarto de miocardio en España. Estudio PREVESE. Rev Esp Cardiol 1997; 50: 406-415.

7. EUROASPIRE Study Group. EUROASPIRE: a European Society of Cardiology survey of secondary prevention of coronary disease, principal results. Eur Heart J 1997; 18: 1569-1582.

8. EUROASPIRE II Group. Lifestyle and risk factor management and use of drug therapies in coronary patients from 15 countries: pricipal results from EUROASPIRE II. Eur Heart J 2201; 22: 554-572.

9. Abookire SA, Karson AS, Fiskio J, Bates DW. Use and monitoring of "statin" lipid-lowering drugs compared with guidelines. Arch Intern Med 2001; 161: 53-58

10. Holt N, Johnson A, de Belder M. Patient empowerment in secondary prevention of coronary heart disease. Lancet 2000; 356: 314. 